\title{
ON STRONGLY SUM DIFFERENCE QUOTIENT LABELING OF ONE-POINT UNION OF GRAPHS, CHAIN AND CORONA GRAPHS
}

\author{
BY
}

\author{
A.D. AKWU
}

\begin{abstract}
In this paper we study strongly sum difference quotient labeling of some graphs that result from three different constructions. The first construction produces onepoint union of graphs. The second construction produces chain graph, i.e., a concatenation of graphs. A chain graph will be strongly sum difference quotient graph if any graph in the chain, accepts strongly sum difference quotient labeling. The third construction is the corona product; strongly sum difference quotient labeling of corona graph is obtained.

Mathematics Subject Classification 2010: 05B15, 05C38, 20 N05.

Key words: graph labeling, chain graphs, corona graphs, one-point union of graphs, complete graphs.
\end{abstract}

\section{Introduction}

Graph theory terminology and notation are taken from [3]. A labeling of a graph $G$ is a one-to-one mapping from the vertex set of $G$ into a set of integers. Labeled graphs serve as useful mathematical models for a broad range of applications such as coding theory, $x$-ray crystallographic analysis, circuit design, communication network et cetera. By a graph we mean a finite, undirected, connected graph without loops or multiple edges. The concept of strongly sum difference quotient graphs was introduced by ADIGA and SWAmy [1].

Definition 1.1. A graph with $n$ vertices is said to be strongly sum difference quotient (SSDQ) graph if its vertices can be labeled with integers $1,2, \ldots, n$ such that the sum difference quotient function $f_{s d q}$ is injective, i.e., 
the values $f_{s d q}(e)$ on the edges are all distinct. We define the sum difference quotient function $f_{s d q}: E(G) \rightarrow Q$ by $f_{s d q}(e)=\left|\frac{f(v)+f(w)}{f(v)-f(w)}\right|$, if $e$ join vertex $v$ and vertex $w$.

Definition $1.2([2])$. Given two graphs $G$ and $H$, the corona (crown) of $G$ with $H$, denoted by $G \odot H$, is the graph with

$$
\begin{aligned}
& V(G \odot H)=V(G) \cup \bigcup_{i \in V(G)} V\left(H_{i}\right), \\
& E(G \odot H)=E(G) \cup \bigcup_{i \in V(G)} E\left(H_{i}\right) \cup\left\{\left(i, u_{i}\right): i \subset V(G) \text { and } u_{i} \in V\left(H_{i}\right)\right\} .
\end{aligned}
$$

In other words, a corona graph is obtained from two graphs $G$ and $H$, taking one copy of $G$, which is supposed to have order $p$, and $p$ copies of $H$, and then joining by an edge the $k^{t h}$ vertex of $G$ to every vertex in the $k^{t h}$ copy of $H$. An special kind of corona graph is given by $C_{n} \odot K_{1}$, i.e., a cycle with pendant points.

Definition $1.3([5])$. A graph $G$ in which a vertex is distinguished from other vertices is called a rooted graph and the vertex is called a root of $G$.

In Section 2, we give a strongly sum difference quotient labeling of onepoint union of graphs while in Section 3 we also give SSDQ labeling of chain graph. In Section 4, we give SSDQ labeling of the corona graph $C_{n} \odot m K_{1}$.

\section{SSDQ labelings of one-point union of graphs}

Let $G$ be a rooted graph, the graph $G^{(n)}$ obtained identifying the root of $n$-copies of $G$ is called a one - point union of $n$ copies of $G$.

Theorem 2.1. Let $B_{1}, B_{2}, \ldots, B_{m}$ be graphs that have $S S D Q$ labelings. Then there exists a graph $G$ that is a one-point union of $B_{1}, B_{2}, \ldots, B_{m}$ that accepts a $S S D Q$ labeling.

Proof. Denote by $f_{s}$ the strongly sum difference quotient labeling of graphs $B_{s}, 1 \leq s \leq m$. Without loss of generality, we may assume that $f_{s}$ assigns the integers $1,2, \ldots,\left|B_{s}\right|$ to the vertices of graphs $B_{s}$. For every $1 \leq s \leq m-1$, identify the vertex of $B_{s}$ with label 1 with the vertex of fixed graph $B_{i}$ with label 1, the graph so obtained is one-point union of graphs, denoted by $G$. The order of $G$ is $1-m+\sum_{s=1}^{m}\left|B_{s}\right|, 1 \leq s \leq m$ 
Now we shall obtain the $S S D Q$ labeling of $G$. With the above assumption, $B_{s}$ and $B_{i}$ share the vertex labeled 1 , which will be the common vertex between them. After labeling the common vertex, list the remaining vertices (without common vertex) of the graph $G$ with the labels assigned to them by $f_{s}$ in ascending order, that is $k_{q}, k \geq 2$. $q$ is the number of times integer $k$ appears in $G$. Then define a function $\phi$ on the vertices of $G$ (without the common vertex) as follows: $\phi\left(k_{q}\right)=m-1+p, 1 \leq p \leq q$, for $k=2$. Now for $k>2, \phi\left((k)_{q}\right)=\phi\left(k_{p^{\prime}}\right)+p, 1 \leq p \leq q$, where $\phi\left(k_{p^{\prime}}\right)=\phi\left((k-1)_{q}\right)$ for $p=q$. Using the labeling $\phi$ on the vertices of $G$, we have a $S S D Q$ labelings of $G$.

Example. Consider the graphs $B_{1}=C_{3}, B_{2}=S_{5}, B_{3}=P_{4}, B_{4}=C_{4}$ with the following labels in Figure 1.
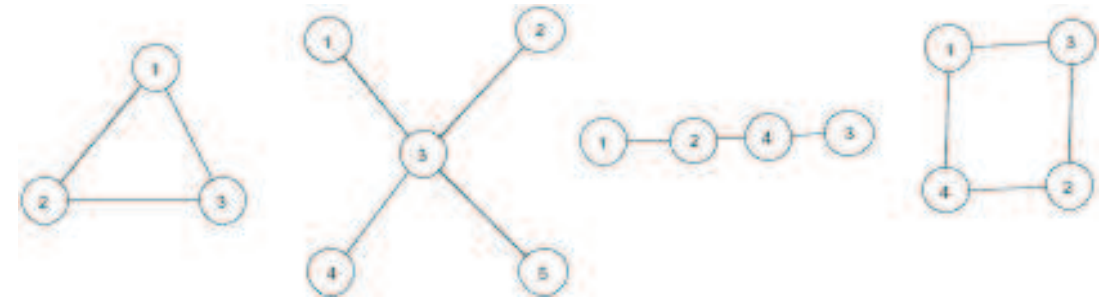

Figure 1: $B_{1}=C_{3}, B_{2}=S_{5}, B_{3}=P_{4}, B_{4}=C_{4}$

In Figure 2 we show a one-point union graph $G$ obtained by using $B_{1}, B_{2}, \ldots, B_{m}$ where $B_{2}$ is fixed. The vertex label of $G$ is a strongly sum difference quotient $(S S D Q)$ graph.

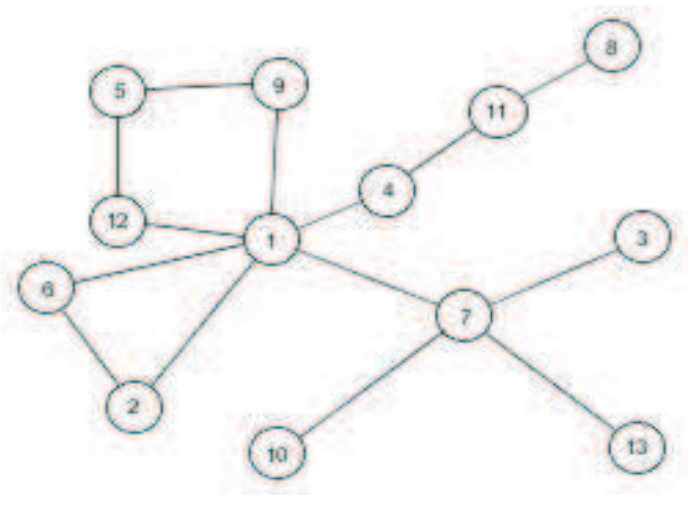

Figure 2: one - point union graph $G$ 


\section{SSDQ labelings of chain graphs}

Suppose now, that the graphs $B_{1}, B_{2}, \ldots, B_{m}$ are blocks and that for every $i \in\{1,2, \ldots, m\}, B_{i}$ and $B_{i+1}$ have a vertex in common, in such a way that the block-cut point graph is a path. The graph $G$ obtained from this concatenation will be called a chain graph.

Let $G$ be a chain graph with $m$ blocks $(m \geq 3)$. Let $u_{i}$ be the vertex that connect $B_{i}$ to $B_{i+1}, 1 \leq i \leq m-1$. So $\left\{u_{i}, u_{2}, \ldots, u_{m-1}\right\}$ is the set of cut vertices of $G$. Let $d_{i}=d\left(u_{i}, u_{i+1}\right)$, namely, the distance between $u_{i}$ and $u_{i+1}, 1 \leq i \leq m-2$. Therefore, each chain graph $G$ has associated the string $d_{1}, d_{2}, \ldots, d_{m-2}$.

Let $G$ be any chain graph with blocks $B_{1}, B_{2}, \ldots, B_{m}$ such that $B_{i}$ is any complete bipartite graph. Then $G$ has a string $d_{1}, d_{2}, \ldots, d_{m-2}$ where $d_{i} \in\{1,2\}$. In this case, it is possible to obtain $S S D Q$ labeling of $G$.

Lemma 3.1. The complete bipartite graph $K_{n, n}$ is $S S D Q$ graph if and only if $n \leq 2$.

Proof. Assume that $K_{n, n}$ is $S S D Q$ graph. We now show that $K_{n, n}$ is not $S S D Q$ for any $n \geq 3$. On contrary, let us assume that $K_{n, n}$ is $S S D Q$ graph for some $n \geq 3$. Let $\{X, Y\}$ be a partition of the graph $K_{n, n}$, where $n \geq 3$. It is clear that $|X|=|Y|=n$, labels assigned to $X$ and $Y$ are distinct and the union of labels assigned to $X$ and $Y$ is $\{1,2, \ldots, 2 n\}$.

Let $x, y$ be labels assigned to the vertices of $K_{n, n}$, if $x=1$ and $y=2$, where $x \in X$ and $y \in Y$ then

$$
f_{s d q}(x, y)=f_{s d q}(2 y, y)
$$

which implies that labels $y, 2 y \in Y$. Also

$$
f_{s d q}(x, y)=f_{s d q}(2 x, 2 y), x, y \in n .
$$

This implies that labels $y, 2 y, 2 x \in Y$. Lastly,

$$
f_{s d q}\left(x_{i}, y_{i}\right)=f_{s d q}\left(x_{j}, y_{j}\right), 1 \leq i, j \leq n,
$$

whenever $y_{i}=2 x_{i}$ and $y_{j}=2 x_{j}$.

Combining equations (3.1), (3.2) and (3.3), we have the labels assigned to vertices in $Y$ to be $\{2,4,3,6, \ldots, 2 n\}$. This implies that $|Y|>n$, a contradiction. Therefore $n \leq 2$. 
Conversely, let $n \leq 2$ and we need to prove that for $K_{n, n}$ is a $S S D Q$ graph. The case $n=1$ is trivial so we prove for the case $n=2$.

If $n=2$, then the number of edges in $K_{n, n}$ is 4 . Assume that vertex labeled with label $x, 2 x$ are not in the same partite set. Then $f_{s d q}\left(x_{1}, y_{1}\right) \neq$ $f_{s d q}\left(x_{1}, y_{2}\right) \neq f_{s d q}\left(x_{2}, y_{1}\right) \neq f_{s d q}\left(x_{2}, y_{2}\right)$. Therefore $K_{n, n}$ is a $S S D Q$ graph when $n \leq 2$.

Theorem 3.1. Let $B_{1}, B_{2}, \ldots, B_{m}$ be blocks of complete bipartite graph $K_{2,2}$ with $S S D Q$ labeling, then any chain graph $G$, obtained by the concatenation of these blocks, has a SSDQ labeling.

Proof. Let $d_{1}=d_{2}=\ldots=d_{m-2}=2$ the string associated to $G$, where the set of cut-vertices $u_{i} 1 \leq i \leq m-1$ of $G$ are not adjacent.

Let $B_{i}=k_{x_{i}, y_{i}} 1 \leq i \leq m$ with bipartite sets $X_{i}$ and $Y_{i}$ of order $x_{i}$ and $y_{i}$ respectively. In the construction of the desired labeling, the cut-vertices between $B_{i}$ and $B_{i+1}$ belongs to $X_{i}$ and $X_{i+1}$. The vertices of the cut-vertex $u_{1}, u_{2}, \ldots, u_{m-1}$ between block $B_{i}$ and $B_{i+1} 1 \leq i \leq m-i$ are labeled as $2 i+1$. The vertices of $X_{1}$ are labeled with the integers $1, u_{1}$ and the vertices of $Y_{1}$ are labeled with the integers $u_{1}-1=2, q$ where $q=\sum_{i=1}^{m} x_{i} y_{i}-m+1$. The vertices of $X_{2}$ are labeled with the integers $3=u_{1}, 5=u_{2}$ and the vertices of $Y_{2}$ are labeled with the integers $q-1, u_{1}+1$.

Since $d_{i}=2$, the vertices of $X_{i+2}$ are labeled with the integers $u_{i}+1, u_{i}+$ 2 and the vertices of $Y_{i+2}$ are labeled with the integers $u_{i+2}-1, q-(i+1)$ such that they are adjacent to the vertices of $X_{i+2}$. Suppose there exist an edge $x_{i} y_{i}$ in $G$ such that the label of vertex $x_{i}$ is a multiple of $y_{i}$ or vice-versa (except edge $x_{1} y_{1}$ ), then replace the label of the vertex $y_{i}$ with the label of the vertex $y_{i-1}$ and vice-versa.

Also, suppose there exist edges $x_{i} y_{i}$ and $x_{i}^{\prime} y_{i}^{\prime}$ in $G$ such that the label of vertex $x_{i}^{\prime}$ is a multiple of the label of the vertex $x_{i}$ and the label of vertex $y_{i}^{\prime}$ is a multiple of the label of the vertex $y_{i}$, then replace the label of the vertex $y_{i}^{\prime}$ with the label of the vertex $y_{i-2}$ and vice-versa. Note that for every block $B_{i}(1 \leq i \leq m)$, the numbering obtained is a $S S D Q$ labeling. This complete the proof.

\section{SSDQ labelings of corona graphs}

An special kind of corona graph is given by $C_{n} \odot K_{1}$, i. e., a cycle with pendants points. In 1979, FRUCHT [4] shown that this corona graph is graceful. Following Frucht's idea, BARRIEntos [2] obtained that any cycle 
with $m$ pendant points attached $(m \geq 1)$ is graceful. We obtained the $S S D Q$ labelings of the corona $C_{n} \odot m K_{1},(m \geq 1)$.

Theorem 4.1 ([1]). For all $n \geq 3$, the cycle $C_{n}$ is a SSDQ graph.

Theorem 4.2. The corona $C_{n} \odot m K_{1}$ is a $S S D Q$ graph for every positive integers $n \geq 3$ and $m \geq 1$.

Proof. Describe the cycle by the circuit $v_{1}, v_{2}, \ldots, v_{n}, v_{1}$ and denote by $v_{i j}$ the vertices of degree one adjacent to vertex $v_{i},(1 \leq i \leq n)$ and $1 \leq j \leq m)$. We distinguish two cases:

Case 1. $n$ is even, the labeling $f$ of $C_{n} \odot m K_{1}$ is define below:

$$
\begin{aligned}
& f\left(v_{2 k+1}\right)=(m+1) k+1, \quad 0 \leq k \leq \frac{n}{2}-1, \\
& f\left(v_{2 k}\right)=(m+1)(n-k)+1, \quad 1 \leq k \leq \frac{n}{2},
\end{aligned}
$$

and $f\left(v_{i, j}\right)=(m+1) n-f\left(v_{i}\right)+(2-j), 1 \leq i \leq n, 1 \leq j \leq m$.

Case 2. $n$ is odd, the labeling $f$ of $C_{n} \odot m K_{1}$ is define below:

$$
\begin{aligned}
& f\left(v_{2 k+1}\right)=(m+1) k+1,0 \leq k \leq \frac{n-1}{2}, \\
& f\left(v_{2 k}\right)=(m+1)(n-k)+1,1 \leq k \leq \frac{n-1}{2},
\end{aligned}
$$

and $f\left(v_{i, j}\right)=(m+1) n-f\left(v_{i}\right)+(2-j), 1 \leq i \leq n, 1 \leq j \leq m$. Now we need to show that labeling $f$ of the corona graph satisfies the required properties of $S S D Q$ graph. The number of vertices in $C_{n} \odot m K_{1}$ is $n(m+1)$. From the labeling $f$ above, the vertices of the corona are labeled with integers $1,2, \ldots, n(m+1)$.

Let $f_{s d q}\left(e_{1}\right)=\frac{\left|f\left(v_{2 k+1}\right)+f\left(v_{2 k}\right)\right|}{\left|f\left(v_{2 k+1}\right)-f\left(v_{2 k}\right)\right|}=\frac{|(m+1) n+2|}{|(m+1)(2 k-n)|}, 0 \leq k \leq \frac{n}{2}$, for $n$ odd and $0 \leq k \leq \frac{n-1}{2}$, for $n$ even.

Let $f_{s d q}\left(e_{2}\right)=\frac{\left|f\left(v_{2 k+1}\right)+f\left(v_{i, j}\right)\right|}{\left|f\left(v_{2 k+1}\right)-f\left(v_{i, j}\right)\right|}=\frac{\left|(m+1)(k+n)-f\left(v_{i}\right)+(2-j)+1\right|}{\left|(m+1)(k-n)+f\left(v_{i}\right)-(2-j)+1\right|}, 0 \leq k \leq \frac{n}{2}$, for $n$ odd and $0 \leq k \leq \frac{n-1}{2}$, for $n$ even, $1 \leq i \leq n, 1 \leq j \leq m$.

Note that $1 \leq f\left(v_{i}\right) \leq n(m+1)$ since $f\left(v_{i}\right)$ is the label assigned to the vertex $v_{i}$. Also $1 \leq j \leq m$ and $k \leq \frac{n}{2}$, then we have $f_{s d q}\left(e_{2}\right) \leq$ $\frac{\left|(m+1) \frac{n}{2}-m+3\right|}{\left|(m+1) \frac{n}{2}+m-1\right|}$. Let

$$
f_{s d q}\left(e_{3}\right)=\frac{\left|f\left(v_{2 k}\right)+f\left(v_{i, j}\right)\right|}{\left|f\left(v_{2 k}\right)-f\left(v_{i, j}\right)\right|}=\frac{\left|(m+1)(2 n-k)-f\left(v_{i}\right)+(2-j)+1\right|}{\left|f\left(v_{i}\right)-(m+1) k-(2-j)+1\right|} .
$$


Note that $1 \leq f\left(v_{i}\right) \leq n(m+1)$ since $f\left(v_{i}\right)$ is the label assigned to the vertex $v_{i}$. Also $1 \leq j \leq m$ and $k \leq \frac{n}{2}$. Then we have $f_{s d q}\left(e_{3}\right) \leq \frac{\left|(m+1) \frac{n}{2}-m+3\right|}{\left|(m+1) \frac{n}{2}+m+1\right|}$. Now to show that all edges of the corona graph are distinct for all values of $k, i$ and $j$, i.e., $f_{s d q}\left(e_{1}\right) \neq f_{s d q}\left(e_{2}\right) \neq f_{s d q}\left(e_{3}\right)$.

Let $f_{s d q}\left(e_{1}\right)=f_{s d q}\left(e_{2}\right), \frac{\left|f\left(v_{2 k+1}\right)+f\left(v_{2 k}\right)\right|}{\left|f\left(v_{2 k+1}\right)-f\left(v_{2 k}\right)\right|}=\frac{\left|f\left(v_{2 k+1}\right)+f\left(v_{i, j}\right)\right|}{\left|f\left(v_{2 k+1}\right)-f\left(v_{i, j}\right)\right|}$. By simplifying the above equation, we have

$$
\frac{|(m+1) n+2|}{|(m+1)(2 k-n)|} \leq \frac{\left|(m+1) \frac{n}{2}-m+3\right|}{\left|(m+1) \frac{n}{2}+m-1\right|}
$$

By simplifying inequality (4.1), we have

$$
|(m+1) n+2| \leq 0 .
$$

Inequality (4.2) is not true since $|x| \geq 0$ for any integer $x$. Therefore $f_{\text {sdq }}\left(e_{1}\right) \neq f_{\text {sdq }}\left(e_{2}\right)$.

Also, let $f_{s d q}\left(e_{2}\right)=f_{s d q}\left(e_{3}\right)$ and assume that

$$
\frac{|(m+1) k-m+3|}{|(m+1) k+m-1|}=\frac{|(m+1)(n-k)-m+3|}{|(m+1)(n-k)+m-1|} .
$$

Inequality (4.3) is not true since $n-k$ is a positive integer greater than $k$. Therefore inequality (4.3) is not true. Hence $f_{s d q}\left(e_{2}\right) \neq f_{s d q}\left(e_{3}\right)$.

Now to show that $f_{s d q}\left(e_{1}\right) \neq f_{s d q}\left(e_{3}\right)$. Let

$$
f_{s d q}\left(e_{1}\right)=f_{s d q}\left(e_{3}\right), \frac{\left|f\left(v_{2 k+1}\right)+f\left(v_{2 k}\right)\right|}{\left|f\left(v_{2 k+1}\right)-f\left(v_{2 k}\right)\right|}=\frac{\left|f\left(v_{2 k}\right)+f\left(v_{i, j}\right)\right|}{\left|f\left(v_{2 k}\right)-f\left(v_{i, j}\right)\right|} .
$$

By simplifying the above equation, we have

$$
\frac{|(m+1) n+2|}{|(m+1)(2 k-n)|} \leq \frac{\left|(m+1) \frac{n}{2}-m+3\right|}{\left|(m+1) \frac{n}{2}+m+1\right|}
$$

By simplifying equation (4.4), we have

$$
|(m+1) n+2| \leq 0 .
$$

Inequality (4.5) is not true since $|x| \geq 0$ for any integer $x$. Therefore $f_{s d q}\left(e_{1}\right) \neq f_{s d q}\left(e_{3}\right)$. Hence $f_{s d q}\left(e_{1}\right) \neq f_{s d q}\left(e_{2}\right) \neq f_{s d q}\left(e_{3}\right)$ and the edges satisfy $f_{s d q}: E(G) \rightarrow Q$. Therefore the labeling $f$ is a $S S D Q$ labeling of $C_{n} \odot m K_{1}$ and the corona graph is a $S S D Q$ graph. 
Example. To avoid an excessive number of figures, we represent the labelings of $C_{n} \odot m K_{1}$ in an array of $n$ columns and $m+1$ rows. The first row containing the labels of vertices of the cycle $C_{n}$; from the second row of any column, are the labels of the end vertices as we can see below.

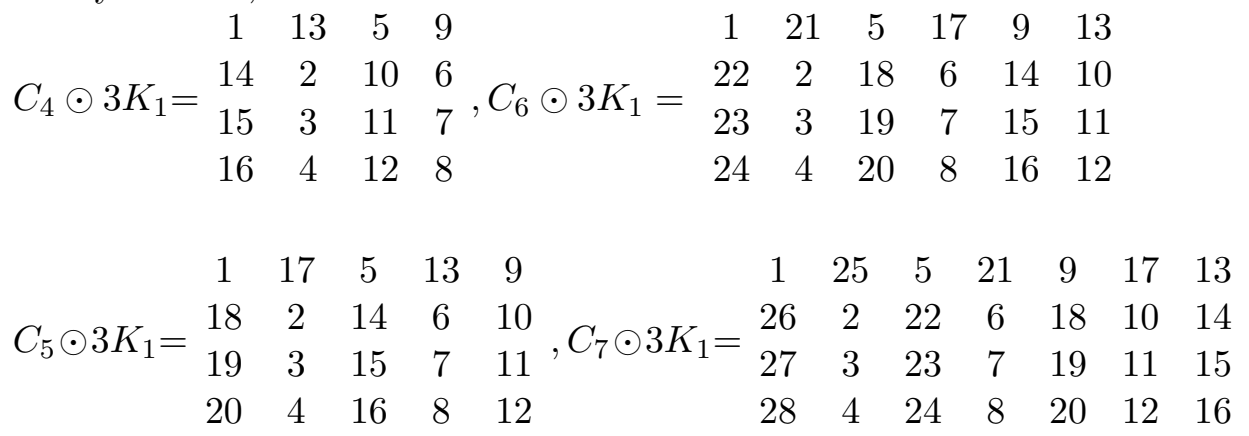

\section{REFERENCES}

1. Adiga, C.; Swamy, C.S. - Shivakumar On strongly sum difference quotient graphs, Adv. Stud. Contemp. Math. (Kyungshang), 19 (2009), 31-38.

2. Barrientos, C. - Graceful labelings of chain and corona graphs, Bull. Inst. Combin. Appl., 34 (2002), 17-26.

3. Chartrand, G.; Lesniak, L. - Graphs and Digraphs, Second edition. The Wadsworth \& Brooks/Cole Mathematics Series. Wadsworth \& Brooks/Cole Advanced Books \& Software, Monterey, CA, 1986.

4. FRUCht, R. - Graceful numbering of wheels and related graphs, Second International Conference on Combinatorial Mathematics (New York, 1978), pp. 219-229, Ann. New York Acad. Sci., 319, New York Acad. Sci., New York, 1979.

5. SheE, S.C.; Ho, Y.S. - The cordiality of one-point union of $n$ copies of a graph, Discrete Math., 117 (1993), 225-243.

Received: 10.III.2012

Revised: 15.VI.2012

Revised: 19.IX.2012

Revised: 6.XI.2012

Accepted: 22.XI.2012
Department of Mathematics, Federal University of Agriculture, Makurdi, NIGERIA

abolaopeyemi@yahoo.co.uk 